\title{
Novel sensing approach for LPG leakage detection: Part I: Operating Mechanism and Preliminary Results
}

\author{
Anindya Nag, Asif I. Zia, Li Xie, Subhas Chandra \\ Mukhopadhyay \\ School of Engineering and Advanced Technology \\ Massey University (Manawatu Campus) \\ Palmerston North, New Zealand
}

\author{
Jürgen Kosel \\ Sensing, Magnetism and Microsystems Group, \\ King Abdullah University of Science and Technology, \\ Saudi Arabia
}

\begin{abstract}
Gas sensing technology has been among the topical research work for quite some time. This paper showcases the research done on the detection mechanism of leakage of domestic cooking gas at ambient conditions. MEMS-based interdigital sensors were fabricated on oxidized single crystal silicon surfaces by maskless photolithography technique. The electrochemical impedance analysis of these sensors was done to detect liquefied petroleum gas (LPG) with and without coated particles of tin oxide $\left(\mathrm{SnO}_{2}\right)$ in form of thin layer.A thin-film of $\mathrm{SnO}_{2}$ was spin-coated on the sensing surface of the interdigital sensor to induce selectivity to LPG that consists of a 60/40 mixture of propane and butane respectively. The paper reports a novel strategy for gas detection under ambient temperature and humidity conditions. The response time of the coated sensor was encouraging and own a promising potential to the development of a complete efficient gas sensing system.
\end{abstract}

Keywords-Interdigital sensors, LPG, electrochemical impedance spectroscopy, coating.

\section{INTRODUCTION}

Liquefied Petroleum Gas (LPG) had been used for last six decades, initially as an industrial fuel [1] and then for domestic cooking [2] and heating purposes [3] due to its smokeless burning in air. The main constituent of this gas is propane and butane mixed in a ratio of 60:40 normally [4]. However, the proportion of the two main gases may differ depending on specified application as well as seasonal condition. Gas leakage detection in residential houses has become one of the fundamental issues in recent times. Electronic and press media have reported some accidents caused due to gas leakage in residential houses $[5,6]$ and in industrial sectors $[7,8]$. Usually, the accidents happen due to negligence rather than technical fault that cause gas to escape into the air.

The paper was submitted on October 3, 2015.

The authors are with the School of Engineering and Advanced Technology, Massey University, Palmerston North, New Zealand. The email of the corresponding author is S.C.Mukhopadhyay@massey.ac.nz
A better sensing system needs to be developed with enhanced limit of low concentration of gas to reduce accidents caused due to gas leakage. The gas is stored in pressurized metallic cylindrical bottles because its boiling point is lower than the ambient temperature. The gas is highly inflammable and is molecularly heavier than any other gas presents the air. Therefore, it settles closest to the ground level and keeps accumulating near the floor in the closed premises unless there is powerful exhaust system operating in the vicinity for its forceful disposal in the open atmosphere.It cannot be detected by human sense of smell due to its odourless nature. A pungent odour chemical is purposely added into the gas to make the presence of this gas detectable by human natural odour sensing system that facilitates human beings to sense its presence without any technical gadget. There are mainly two major drawbacks of such system. Firstly, it requires a human subject present in the vicinity. Secondly, by the time its presence in air could be sensed manually, its concentration exceeds a threshold level that is enough to cause an explosion by any spark triggering device like a light switch or metallic contact friction. In order to monitor its presence, sensors and sensing systems are deployed in LPG installed premises and environments to detect the leakage of gas and to avoid the accidents. There are some commercial gas detecting sensors [9] and systems available in the market that can issue warnings for a gas leakage incident. These sensors work on the concept of fixed controllers, fixed transmitters and fixed accessories. However, the cost of these commercialized sensing systems is high and has moderate technical complexity and inaccuracy with large delay in the response time. Therefore, a need for the development of a lowcomplexity, fast response and low-cost sensing system still exists.

A significant amount of research work has been executed previously by employing different types of sensors for gas detection [10-14]. But, it still is a hot topic of research due to the selectivity and sensitivity issues in the sensing mechanism and above-all long response and recovery time associated with the sensor.The work describes invoking a new methodoly to improve the mentioned attributes.

The paper is divided into five sub-sections. After introduction in Section I, the working principle of the interdigital sensor 
used for gas sensing and the experimental set-up are explained in section II. Results in section III show the response of the sensor towards the gas. The discussion part is given in section IV which explains the mechanism behind gas detection. The conclusion and the future work are given in section $\mathrm{V}$ inferring the work done and further experiments that need to be done to validate of the proposed approach.

\section{INTERDIGITAL SENSOR AND EXPERIMENTAL SET-UP}

2.1 Interdigital sensor: Working principle and fabrication

Interdigital sensors are used for the conduction of experiments. These sensors are based on the operating principle of parallel plate capacitors. The schematic diagram of interdigital sensor is shown in figure 1. The planar structure of the capacitor is formed for one-directional, non-invasive measurements. The planar geometry of the sensor introduces bulging electric field between the two plates in contrary to the generation of the uniformly parallel electric field in a co-axial form. When a time-dependent voltage signal is applied to the excitation, an electric field is generated between the two polarized plates as a result of the potential difference between them. Figure 1 clearly depicts the conversion of the parallel plate capacitor to planar form. The sensor can be used to measure the properties of material under test in many different ways. When any material is kept within the penetration depth of the electric lines of force of the sensing surface, the electric field passes through that material and gets modified by the material under test (MUT). The resultant electric fields which can provide information of the MUT are obtained from the measurement of sensor impedance.
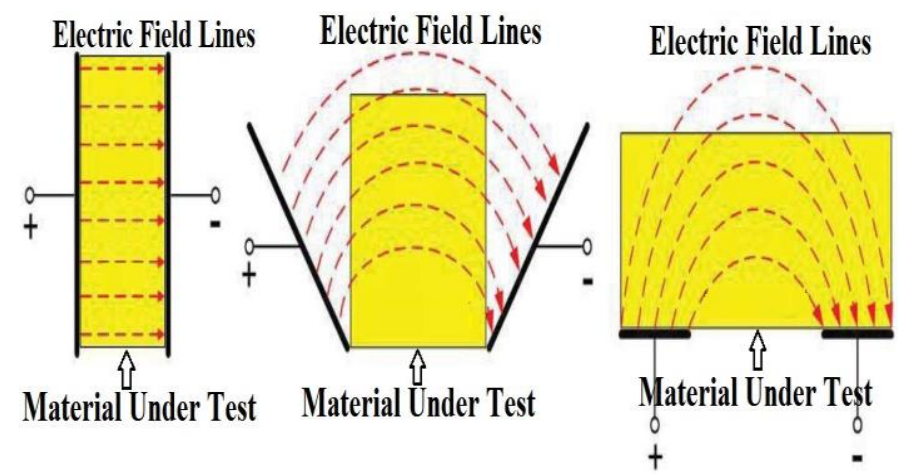

Fig. 1: Schematic diagram of interdigital sensor [15].

The sensor chosen for the experiment was of 1-5-50 configuration. The reason for choosing such a configuration was to have a better resultant electric field from the excitation electrode to the sensing electrode [16].This sensor has a configuration of 1-5-50, implying the presence of five sensing electrodes with respect to one excitation electrode, having a distance of 50 microns between two consecutive electrodes.

For this research, new sensors have been fabricated using maskless photolithography. Single crystal p-type silicon wafers with a thickness of 525 microns were selected for constructing of interdigital sensors. An insulating layer of silicon dioxide was formed by thermal oxidation on these silicon wafers. The thickness of the oxidation layer was 500 nanometers. The wafer was coated with lift-off resistance (LOR) and positive photoresist, ECI 3027 followed by prebaking the wafer at $180^{\circ} \mathrm{C}$ at 7 minutes and $100^{\circ} \mathrm{C}$ for 5 minutes respectively. The designs of the structure of the sensors were done using Windows supported L-Edit software, which was fed into a photolithography device for exposure of the patterns on the wafers. The wafer was then chemically, and plasma etched, followed by sputtering of metals of specified thickness on the etched patterns. The wafer was then sonicated, dried with nitrogen gas and finally cut into individual sensors. The fabricated sensor used for the experiment is shown in figure 2 .

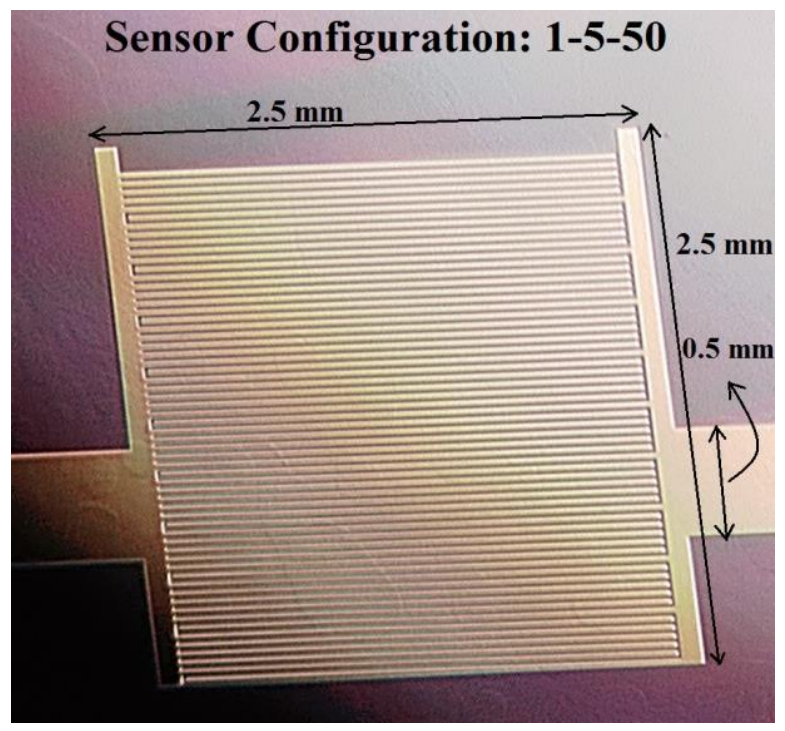

Fig. 2: Interdigital sensor having a configuration of 1-5-50.

To measure the capacitance of the sensor and consequently the dielectric permittivity, help of an electric circuit is taken. Figure 3 shows the equivalent circuit of the sensor along with a series resistance in presence of an electrical signal. The value of the series resistance will be very low so that it does not affect anything on measurement.

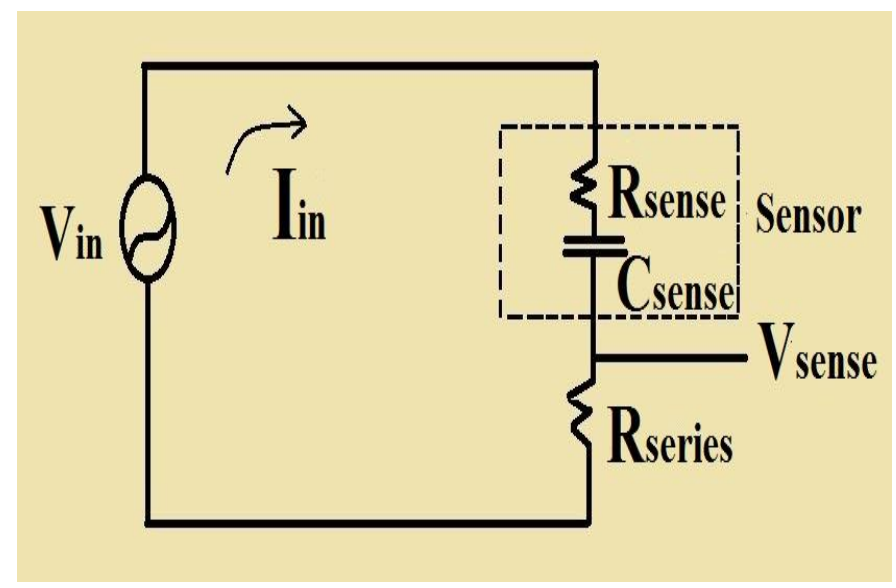

Fig. 3: Equivalent circuit of interdigital sensor. 
The operating principle of the circuit for an interdigital sensor can be explained as follows:

$$
V_{\text {sense }}=I_{\text {in }} * R_{\text {series }}
$$

Where $V_{\text {sense }}$ isthevoltage measured across the series resistance and $I_{\text {in }}$ is the current following through the sensor.

$$
Z_{\text {eq }}=\frac{V_{\text {in }}}{I_{\text {in }}}=\frac{V_{\text {in }}}{V_{\text {sense }}} * R_{\text {series }}
$$

Where $Z_{e q}$ is the equivalent impedance of the circuit and

$V_{\text {in }}$ is the input voltage.

$$
\begin{aligned}
& X_{\text {sense }}=Z * \sin \emptyset \\
& R_{\text {sense }}=Z * \cos \emptyset-R_{\text {series }^{--}} \text {(4) }
\end{aligned}
$$

Where $\varnothing$ is the phase angle between $V_{i n}$ and $I_{i n}$.

$$
\begin{aligned}
& X_{\text {sense }}=w * C_{\text {sense }}-(\text { (5) } \\
& \text { And, } C_{\text {sense }}=\frac{\varepsilon_{0} \varepsilon_{r} A}{d}-\text { (6) }
\end{aligned}
$$

Where

$C_{\text {sense }}=$ capacitance of the sensor in Farads, F.

$\varepsilon_{0}=$ the permittivity of free space $\left(\varepsilon_{0}=8.854 \times 10^{-12} \mathrm{~F} / \mathrm{m}\right)$

$\varepsilon_{r}=$ the relative permittivity or dielectric constant of the material under test $($ vacuum $=1)$.

$\mathrm{A}=$ effective area, square meters.

$\mathrm{d}=$ effective spacing between positive and negative electrode, meters.

Usually the phase angle $\emptyset$ between $V_{\text {in }}$ and $I_{\text {in }}$ is close to $90^{\circ} \mathrm{C}$ for any dielectric material. The resistive part $R_{\text {sense }}$ is usually very small or the change is insignificant. In normal dielectric characterization at low operating frequency, the $X_{\text {sense }}$ is studied.

Electrochemical impedance spectroscopy (EIS) technique was used to determine the response of the interdigital sensors towards different concentrations of LPG. The electrochemical impedance spectroscopy technique owns high sensitivity with the limitation of stable and reversible system in equilibrium. Therefore, for non-stationary systems, instantaneous impedance measurements are mandatory. Electrochemical impedance spectroscopy and interdigital sensors had been used by a number of researchers like phthalate detection [17$20]$ in food, evaluation of nitrate contamination in water [2125], for environmental monitoring [26], for meat fat content inspection [27, 28], moisture content estimation [29] etc. In the current research, the objective of the initial experiment is investigating response of the uncoated sensor to LPG at standard atmospheric condition.

\subsection{Experimental set-up}

All the experiments were conducted under identical temperature, humidity, and $\mathrm{pH}$ boundary condition in controlled laboratory environment. The sensor was connected to Hioki 3522-50 LCR Hi precision tester (Japan) by gold contact clamp pin connector. The spin coating technique described in the subsequent section was performed with a spin coater of 6800 series. The chemicals used for spin coating were brought from Sigma-Aldrich. Tin oxide (IV) had purity of $97 \%$ with a density $6.95 \mathrm{~g} / \mathrm{mL}$ at $25{ }^{\circ} \mathrm{C}$ (lit.). Ethyl alcohol had a purity of $>99.9 \%$ with a flash point at $14^{\circ} \mathrm{C}$.

The LCR meter was initially run with built in open and short calibration tests to nullify any stray capacitance due to connecting leads. LCR tester was set to slow mode of testing to achieve an error rate of $<0.05 \%$ as recommended by the manufacturer. The sensor setup was formed in a desiccator to achieve the required controls of $44 \%$ humidity and inert atmosphere. To obtain a reference, the sensor was profiled in the air for a frequency sweep of $42 \mathrm{~Hz}$ to $2 \mathrm{MHz}$ with ten measurements per decade change in frequency at log scale. No significant change of humidity was noticed during the experiments.

The detection of LPG was carried out inside a fume hood in a $5000 \mathrm{~cm}^{3}$ airtight chamber with an inlet and an outlet. The interdigital sensor, placed inside the chamber, was connected to the LCR meter through a jig with gold-coated pressure contacts. The LCR meter was connected to the laptop through the serial communication port using RS232 interface. Window based data acquisition software was used to write the response signal in a Microsoft Excel file in the computer. The experimental set up is shown in figure 4. The gas used for the experiment was drawn from a $9 \mathrm{~kg}$ pressurized cylindrical bottle. Gas taken from this bottle was mixed with air from two separate tubes to attain a concentration of $20,000 \mathrm{ppm}$, which is the threshold for the explosion. The pressure of the gas and air were controlled at a flow rate of $700 \mathrm{ml} / \mathrm{min}$ and $20 \mathrm{lit} / \mathrm{min}$ by the two rotameters connected in order to achieve the desired concentration of LPG in the air.

The profile for the sensor in air was obtained for the range of real part $(\mathrm{R}(\Omega))$ and the imaginary part of the impedance (X $(\Omega)$ ) to determine the nature of the sensor. The sensor was tested for a range of frequencies from $1 \mathrm{~Hz}$ to $300 \mathrm{~Hz}$ to obtain the optimal frequency of operation for the gas leakage detection. The test frequency for the experiments had been set to $15 \mathrm{~Hz}$.

The characterization of the sensor was done in air and with different concentrations of gas to determine its response. Nyquist plot, i.e., the imaginary part of the impedance versus the real part of the impedance of the sensor for a range of frequencies has been shown in figure 5. It can be seen from the figure that the response of the sensor is quite indifferent to air and LPG. The sensing behavior of conventional interdigital sensor is not observed at all. There is a need to modify the surface of the sensor with some coating to induce sensitivity and selectivity. 


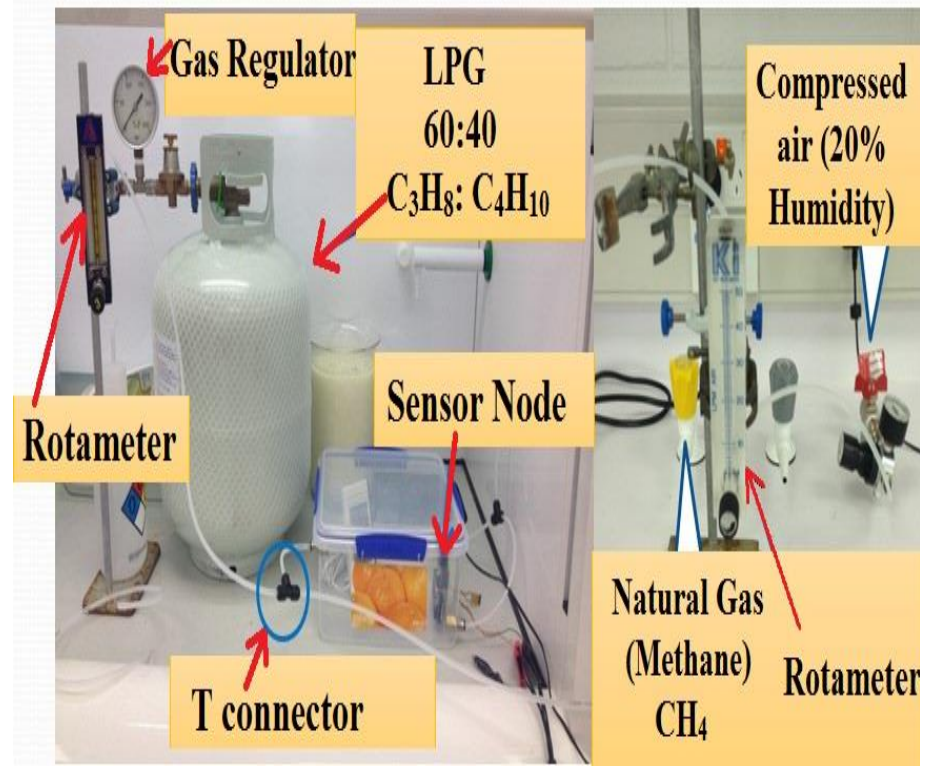

Fig. 4: Experimental set-up for detection of LPG.

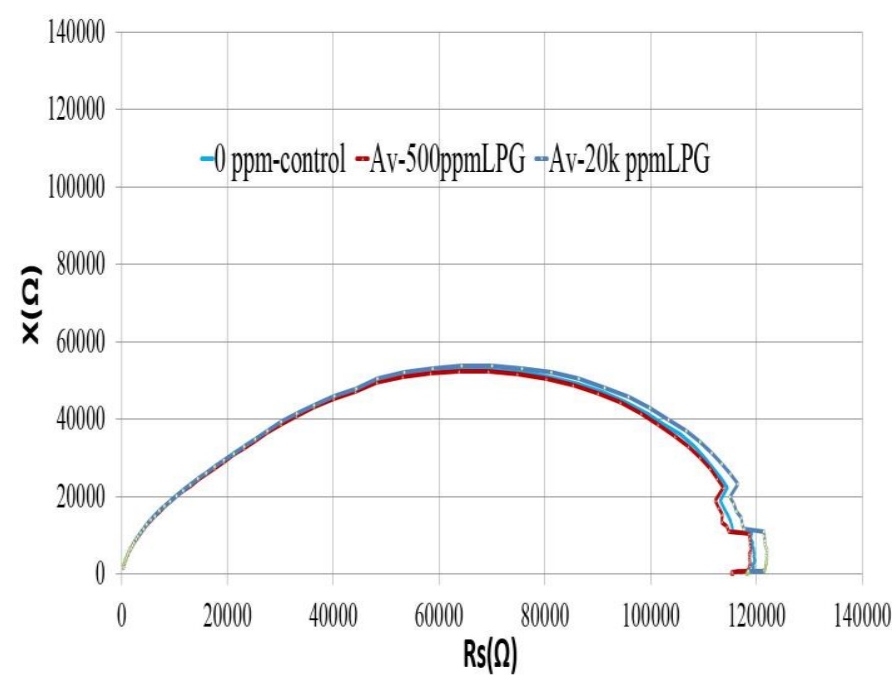

Fig. 5: Response of the sensor without coating towards air and gas.

\subsection{Surface coating: Mechanism and principle}

To induce the sensitivity as well as selectivity of the sensing surface, thin-film coating was done. There are different coating mechanisms available to achieve an LPG selective coating on the sensing surface of the sensor element, such as spin coating [30], dip coating [31] and electrospinning [32]. Spin coating mechanism was chosen among three mentioned coating techniques with the consideration of the following disadvantages of dip coating mechanism and electrospinning. The schematic diagram for spin coating is shown in figure 6 . Firstly, unlike dip coating, spin coating technique forms a uniform layer of coated material. Secondly, the control over the coating thickness could be achieved more easily in comparison to electrospinning methodology. The sensor whose sensing surface was to be coated with a specific coating material was placed on the circular metallic platform. Tin oxide $\left(\mathrm{SnO}_{2}\right)$ and ethyl alcohol has been used as the dispersed and dispersion medium respectively. It was then rotated at fixed revolutions per minute (rpm) and duration to generate a uniform layer over the sensing surface.

The time of spin coating plays a vital part in achieving the target coating thickness. The influence of spinning time was also investigated in this research that is described in detail in later section of the manuscript. The angular speed of the platform that was measured in rpm could be varied to control the coating thickness of the coating. Another factor that affects the coating thickness is the specific gravity of the suspension. These two factors will be investigated and presented in future research work.

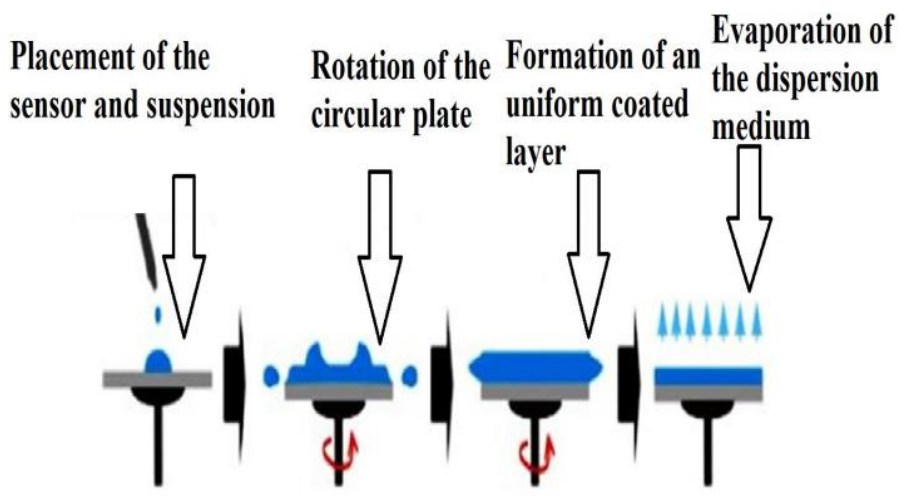

Fig. 6: Schematic diagram for spin-coating process

Numerous oxides have been studied to optimize the conductivity effect. Among them, $\mathrm{SnO}_{2}$ has been regarded as a material with favorable unique properties like chemical and thermal stability, natural non-stoichiometry and a distinct property of better oxygen absorbance.The use of $\mathrm{SnO}_{2}$ has been done in many gas detection $[13,33,34]$ works due to its semiconductive properties. $\mathrm{SnO}_{2}$ was used as a thin-film layer on the sensing surface of the interdigital sensors for the selective LPG detection. The oxidation of the reactive gases at semiconductor metal oxide (SMO) thin film surface induces changes in the electrical conductivity of the film. The absorbed oxygen produce oxide on the surface and formation of oxide in the form of a thin film on the coating surface induce sensitivity, as sensing is essentially a surface phenomenon. Here, an n-type semiconductor is used as a functional layer on the sensing area to induce redox reactions with the gas.The incident molecules of propane and butane get absorbed in the semiconductor coating layer liberated electrons as a result of chemisorption in the thin layer. Hence, a change is observed in the flow of current through the sensor that reflects a change of impedance of the sensor for the material under test.

The suspension was formed with $\mathrm{SnO}_{2}$ using ethyl alcohol as the dispersion medium. A semi-solid porous suspension was formed on mixing the two mentioned compounds. SEM image of the coated sensing surface is shown in figure 7 . The image was obtained from a FEI Quanta 200 model with an 
accelerating voltage of $20 \mathrm{kV}$. The thickness of the coated layer was 2.155 microns. The value was an average of the measurement between the ones taken vertically from the center and the side of the sensing surface by an electron beam directed vertically from the top of the sensor.

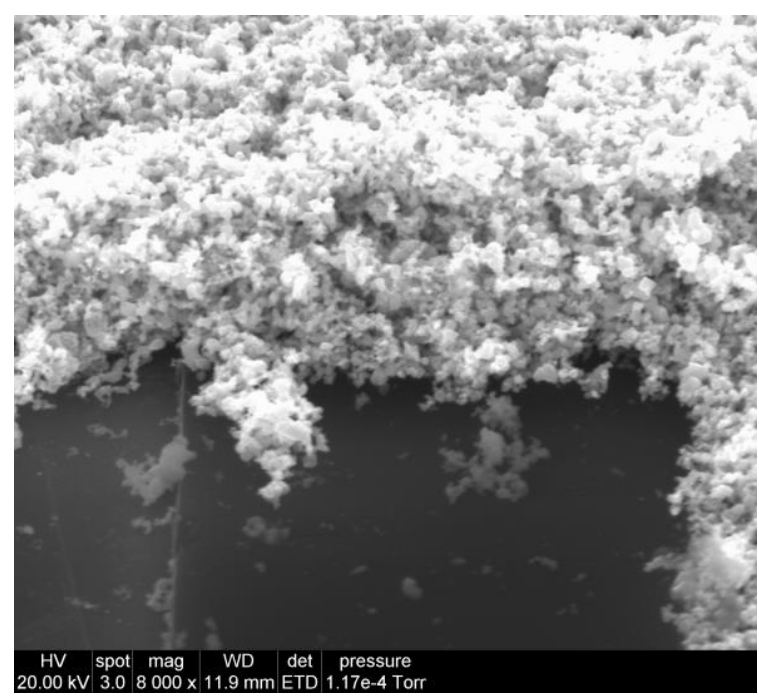

Fig. 7: SEM image of the coated surface.

\section{EXPERIMENTAL RESULTS}

The sensor was tested for different concentrations of gas to determine its response. The gas concentration was varied at a range from $500 \mathrm{ppm}$ to $20,000 \mathrm{ppm}$. Nyquist plot of the sensor for the different concentrations of gas are plotted to analyze the change in impedance with respect to each concentration. The response is shown in figure 8 . It can be seen that there is a change between the impedance values and gas concentration. The highest impedance is corresponding to the lowest concentration inferring to lowest conductivity. As the concentration increases, the impedance changes while principally the resistance keeps on decreasing. It is seen that the change in real part of impedance is more than the imaginary part suggesting that the gas detection is taking place due to the change in conductivity.

The change in impedance, phase angle and real part of the impedance are shown in figures 9, 10 and 11 respectively. The readings are taken at low frequencies from $1 \mathrm{~Hz}-300 \mathrm{~Hz}$ with 20 data points per decade on a logarithmic scale. The reason for considering lower frequencies is due to the charge transfer process that takes place which is explained in the subsequent section. The change in the impedance $(\mathrm{Z})$ is more prominent in lower frequencies compared to higher frequencies. The change in the phase angle $(\Phi)$ is very low at lower frequencies up to an extent (around $10 \mathrm{~Hz}$ ) and then keeps increasing exponentially with increasing frequencies. The real part of the impedance also shows subsequent change in the lower frequencies shown in figure 11. The impedance values changes symmetrically along with the concentration of gas. It can be seen that the resistance values changes almost symmetrically with respect to each concentration of gas. This signifies the change in conductivity occurring at the coated surface of the sensor.

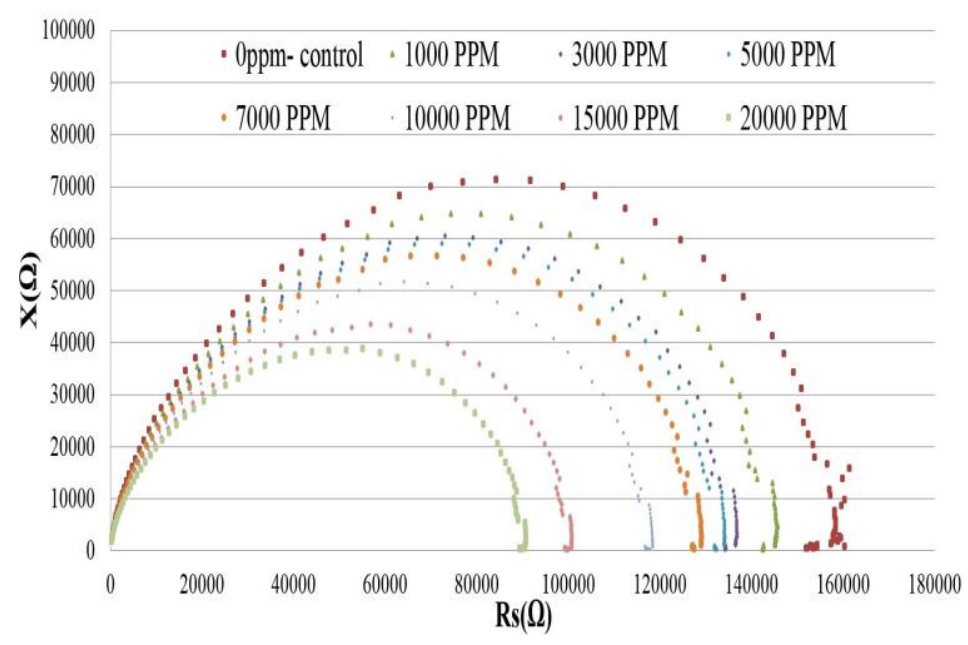

Fig. 8: Response of the sensor with coating towards different concentrations of gas.

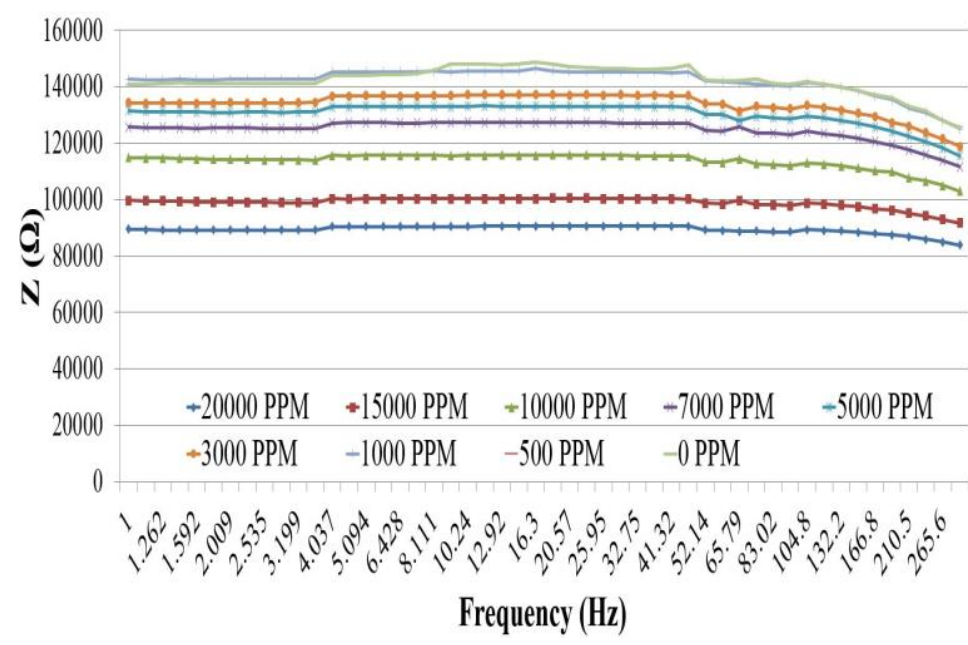

Fig. 9: Change of the impedance $(\mathrm{Z})$ with respect to frequency $(1 \mathrm{~Hz}-300$ $\mathrm{Hz}$ ).

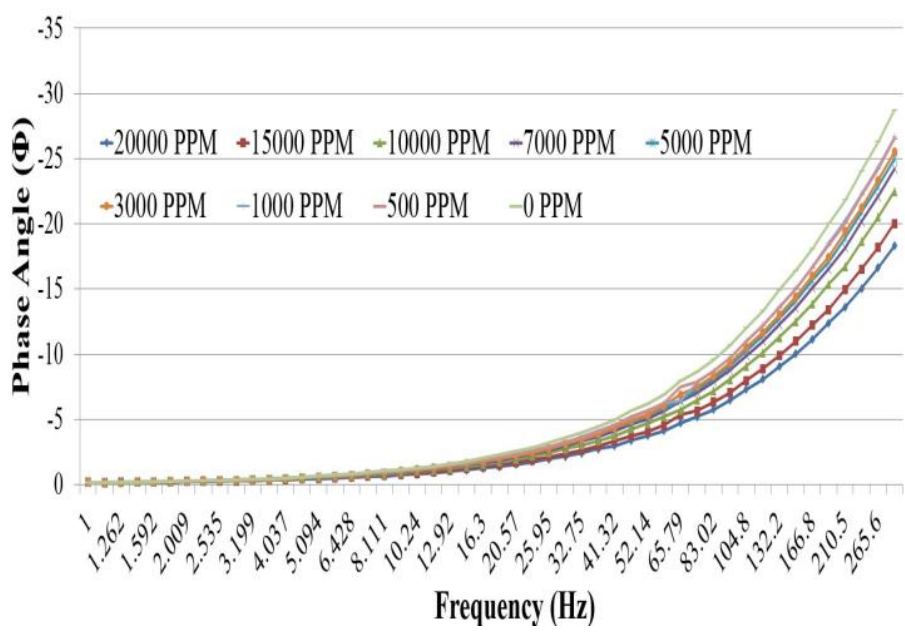

Fig. 10: Change of phase angle $(\Phi)$ with respect to frequency $(1 \mathrm{~Hz}-300 \mathrm{~Hz})$. 
The sensitivity of the real part of impedance towards the gas is shown in figure 12 . The change in $\%$ of sensitivity is calculated from the following formula:

$$
\text { Sensitivity }(\%)=\frac{\left(R_{\text {control }}-R_{\text {measured }}\right)}{R_{\text {control }}} \times 100
$$

It can be seen from figure 12 that the response of resistive values changes almost linearly with the concentration. This signifies that resistive part has a major role to play in the working mechanism of the gas detection.

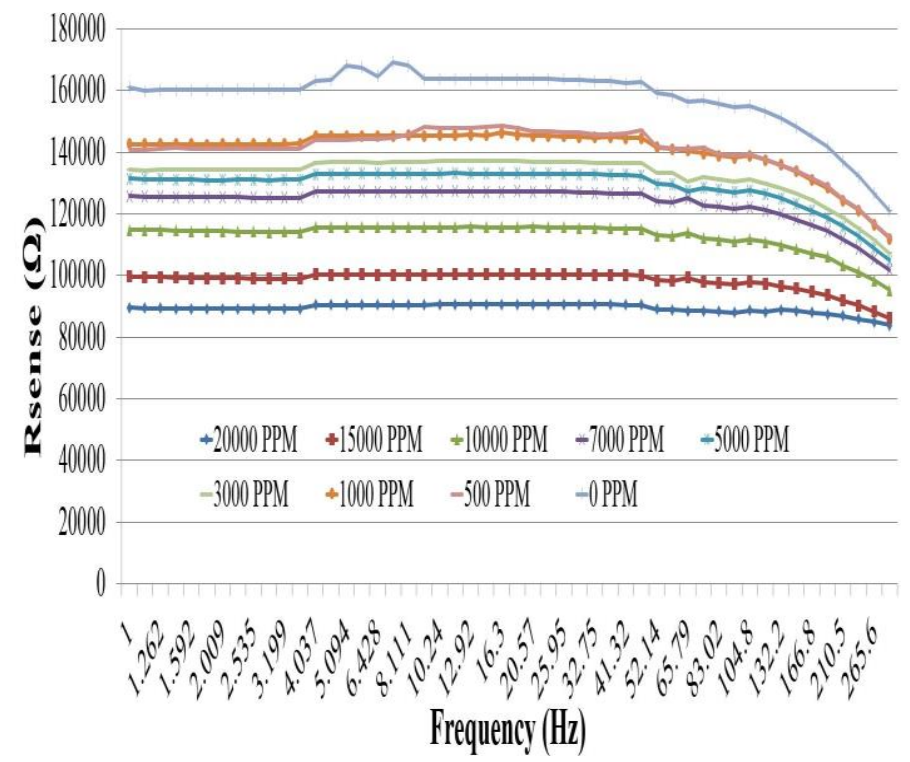

Fig. 11: Change of real part of impedance $(\mathrm{R})$ with respect to frequency (1 $\mathrm{Hz}-300 \mathrm{~Hz})$.

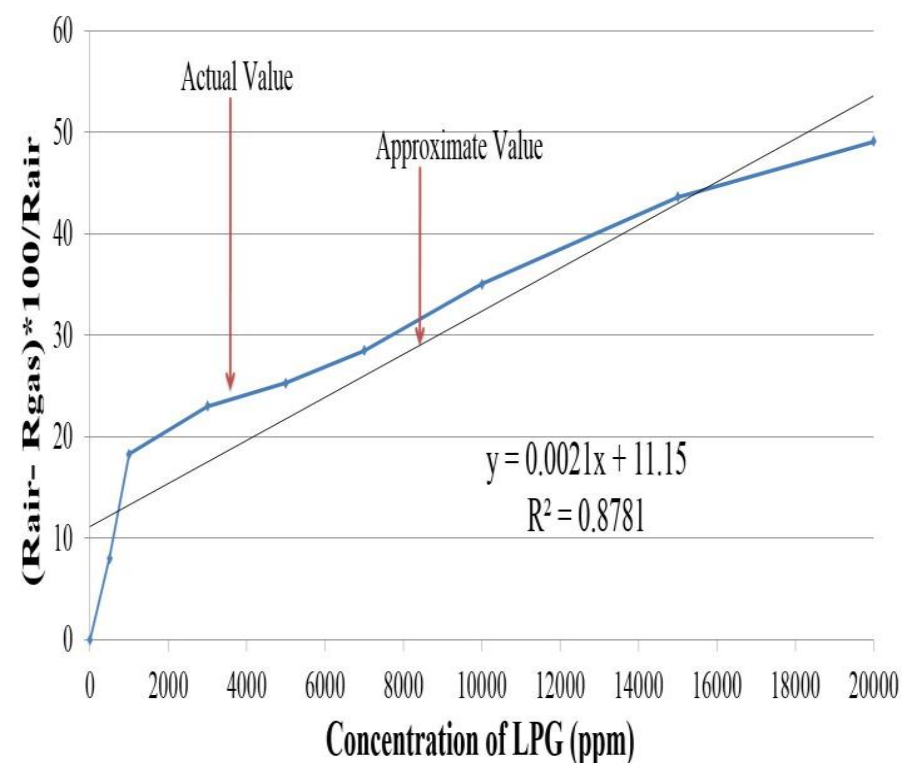

Fig. 12: \% change of sensitivity of $\mathrm{R}$ with respect to different concentrations of gas.

\section{DISCUSSION}

It can be inferred from the above results that the detection of LPG at different concentrations is taking place predominantly due to the change in resistance. A reaction between the coated sensor and the gas has taken place. The kinetics of charge transfer process at lower frequencies is more prominent than the ion diffusion process [35]. This can be explained from the structural change that occurs on reception of the gas. The structure of $\mathrm{SnO}_{2}$ at room temperature is given in figure 13[36]. It can be seen from the lattice structure that the compound is chemically stable in the absence of traces of LPG. LPG undergoes chemisorption when exposed to the interdigital sensors coated with the semiconducting $\mathrm{SnO}_{2}$ thin layer. The resistance of tin oxide thin films changes nonlinearly with the change in the surface temperature, thin film thickness, gas concentration and stoichiometry. A potential barrier mechanism dictates the magnitude of the electrical resistance offered by tin oxide coating in the thin film tin oxide based sensors. The bulk $\mathrm{SnO}_{2}$ under the surface layer behaves as a 'molecular sieve' that prevents the oxygen adsorbed at the surface to diffuse into the bulk and holds it at the grain boundaries that leads to an electron depleted zone at the surface.The hydrocarbon gases such as LPG react with the adsorbed oxygen; consequently increasing the electron density in the $\mathrm{SnO}_{2}$ surface that in turn increases the conductivity of the coating surface. The LPG acts as an oxidizing agent supplying surface oxygen ions to the conduction band of the thin-film coated $\mathrm{SnO}_{2}$ layer, thus increasing the conductivity.

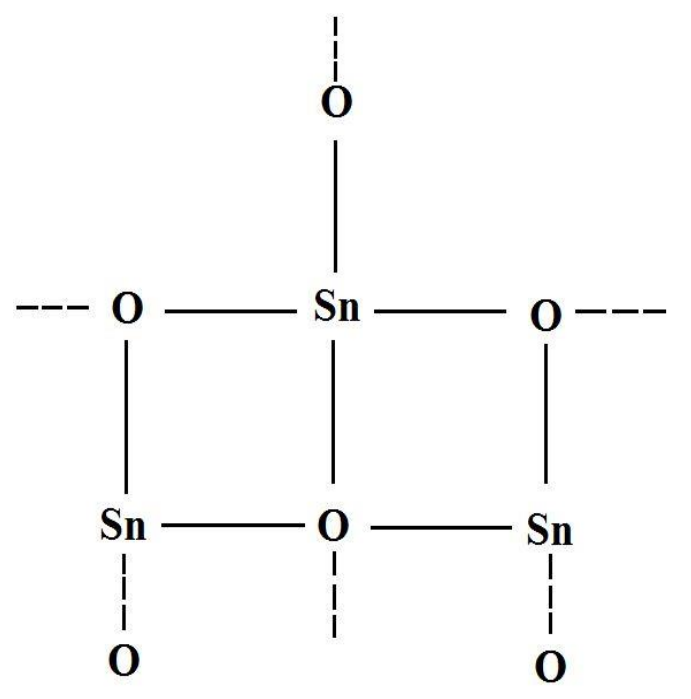

Fig. 13: $\mathrm{SnO}_{2}$ structure at room temperature (normal condition).

Reduction reaction takes place for the long chains of propane and butane contained in LPG. A p-type response was conducted by the $\mathrm{SnO}_{2}$ coating on LPG by attaching to the electrons from the gas resulting in an increase in current flow. The chemisorbed oxygen reacts with the chains of propane and butane leading to the release of electrons. These electrons released by the hydrocarbon chains are attached to the oxygen ions at the surface leading to the formation of a structure 
shown in figure 14. This structure is chemically unstable compared to the normal $\mathrm{SnO} 2$ structure. The inclusion of the electrons in the coated suspension layer signifies the decreases in impedance of the sensor in the presence of LPG. So the real part of the impedance is changing more compared to the imaginary part. But as the input voltage $V_{i n}$ is constant and $R_{\text {sense }}$ is increasing, this will have an effect on $X_{\text {sense }}$ and it won't be constant. But this change will be very nominal compared to $R_{\text {sense }}$.

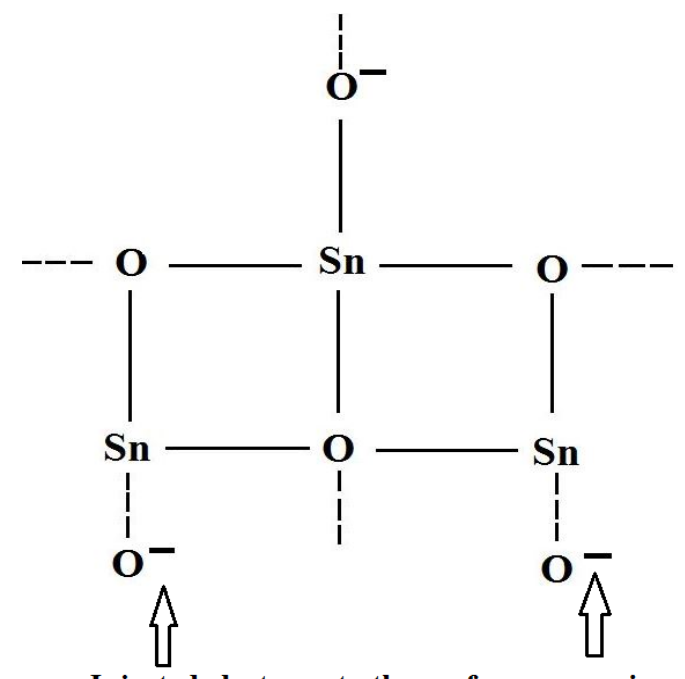

Fig. 14: $\mathrm{SnO}_{2}$ structure formation with $\mathrm{LPG}$

\section{CONLUSION AND FUTURE WORK}

The paper presented a new approach in the detection of LPG at ambient conditions. Temperature and humidity conditions were maintained fixed during experimentation. The fabricated interdigital sensor was used to test different concentrations (in ppm) of LPG under coated and uncoated conditions to analyze the difference in response in two situations. The fabricated interdigital sensor was spin-coated with a thin-film of $\mathrm{SnO}_{2}$ at fixed speed (1300 rpm) and timing (15 seconds) to induce a thin film layer of the sensing surface. The real part of the impedance changes more than the imaginary part as a result of exchange of electrons on the surface oxygen ions of $\mathrm{SnO}_{2}$ on the sensing surface. The sensitivity of the real part of the impedance was calculated to determine the characteristic change in the response of the sensor with the variation of LPG concentration. The sensor showed a substantial change in the conductivity as a result of the exchange of electrons taking place between the gas and coated layer.

The next step would be to test the coated sensor on gas by varying the attributes of the thin-film surface like the concentration of the suspension layer along with the spin coating time and size of the dispersed medium of the suspension. A low-cost, efficient gas sensing system would be developed to be considered for practical applications.

\section{REFERENCES}

[1] J. O. Jaber, "Future energy consumption and greenhouse gas emissions in Jordanian industries," Applied energy, vol. 71, pp. 1530, 2002.

[2] A. D'sa and K. N. Murthy, "Report on the use of LPG as a domestic cooking fuel option in India," International Energy Initiative, Bangalore, pp. 17-19, 2004.

[3] M. Alam, J. Sathaye, and D. Barnes, "Urban household energy use in India: efficiency and policy implications," Energy policy, vol 26, pp. 885-891, 1998.

[4] S. M. Thompson and G. Robertson, "Liquefied Petroleum Gas," Ullmann's Encyclopedia of Industrial Chemistry, 2005.

[5] C. Pietersen, "Analysis of the LPG-disaster in Mexico City," Journal of Hazardous Materials, vol. 20, pp. 85-107, 1988.

[6] S. C. Lee, W.-M. Li, and C.-H. Ao, "Investigation of indoor air quality at residential homes in Hong Kong-case study," Atmospheric Environment, vol. 36, pp. 225-237, 2002. F. Hong, "Xi' an 710054, China); The progress of the technology of geophysical survey to preventing gas accident occurring in the colliery in China [J]," Progress In Geophysics, vol. 4, 2005.

A. Demirbas, "Fuel properties of hydrogen, liquefied petroleum gas (LPG), and compressed natural gas (CNG) for transportation," Energy Sources, vol. 24, pp. 601-610, 2002.

S. Capone, A. Forleo, L. Francioso, R. Rella, P. Siciliano, J. Spadavecchia, et al., "Solid state gas sensors: state of the art and future activities," Journal of Optoelectronics and Advanced Materials, vol. 5, pp. 1335-1348, 2003.

S. Chopra, K. McGuire, N. Gothard, A. Rao, and A. Pham, "Selective gas detection using a carbon nanotube sensor," Applied Physics Letters, vol. 83, pp. 2280-2282, 2003.

J. Li, Y. Lu, Q. Ye, M. Cinke, J. Han, and M. Meyyappan, "Carbon nanotube sensors for gas and organic vapor detection," Nano Letters, vol. 3, pp. 929-933, 2003.

J. Kong, N. R. Franklin, C. Zhou, M. G. Chapline, S. Peng, K Cho, et al., "Nanotube molecular wires as chemical sensors," Science, vol. 287, pp. 622-625, 2000.

Y. Wang, X. Jiang, and Y. Xia, "A solution-phase, precursor route to polycrystalline $\mathrm{SnO} 2$ nanowires that can be used for gas sensing under ambient conditions," Journal of the American Chemical Society, vol. 125, pp. 16176-16177, 2003.

Gas sensing at room temperature. Available: https://books. google.com.sa/books?id=BJmIGor14-

$\mathrm{UC} \& \mathrm{pg}=\mathrm{PA} 158 \& \mathrm{lpg}=\mathrm{PA} 158 \& \mathrm{dq}=$ electrochemical + proceedings + gas+sensors+at+room+temperature $\&$ source $=$ bl\&ots $=3 I u 5$ -

3E6uW\&sig=MJ1JcvoB9pakDz1JGlnfDCObWzA\&hl=en\&sa=X $\&$ redir_esc $=\mathrm{y} \# \mathrm{v}=$ onepage $\& \mathrm{q}=$ electrochemical $\% 20$ proceedings $\% 2$ 0gas\%20sensors\%20at\%20room\%20temperature $\& \mathrm{f}=$ false.

Accessed on 24.10.2015

[15] M. S. A. Rahman, S. C. Mukhopadhyay, and P.-L. Yu, "Novel Planar Interdigital Sensors," in Novel Sensors for Food Inspection: Modelling, Fabrication and Experimentation, ed: Springer, 2014 pp. 11-35.

[16] A. Mohd Syaifudin, S. Mukhopadhyay, and P. Yu, "Electromagnetic field computation using COMSOL Multiphysics to evaluate the performance of novel interdigital sensors," in Applied Electromagnetics Conference (AEMC), 2009, 2009, pp. 1 4.

[17] A. I. Zia, A. Mohd Syaifudin, S. Mukhopadhyay, I. Al-Bahadly, P. $\mathrm{Yu}, \mathrm{C}$. Gooneratne, et al., "Development of Electrochemical Impedance Spectroscopy based sensing system for DEHP detection," in Sensing Technology (ICST), 2011 Fifth International Conference on, 2011, pp. 666-674.

[18] A. I. Zia, S. Mukhopadhyay, I. Al-Bahadly, P. Yu, C. P. Gooneratne, and J. Kosel, "Introducing molecular selectivity in rapid impedimetric sensing of phthalates," in Instrumentation and Measurement Technology Conference (I2MTC) Proceedings, 2014 IEEE International, 2014, pp. 838-843. 
A. I. Zia, M. S. A. Rahman, S. C. Mukhopadhyay, P.-L. Yu, I. AlBahadly, C. P. Gooneratne, et al., "Technique for rapid detection of phthalates in water and beverages," Journal of Food Engineering, vol. 116, pp. 515-523, 2013.

[20] A. I. Zia, A. M. Syaifudin, S. Mukhopadhyay, P. Yu, I. AlBahadly, C. P. Gooneratne, et al., "Electrochemical impedance spectroscopy based MEMS sensors for phthalates detection in water and juices," in Journal of Physics: Conference Series, 2013, p. 012026.

[21] M. A. M. Yunus, G. R. Mendez, and S. C. Mukhopadhyay, "Development of a low cost system for nitrate and contamination detections in natural water supply based on a planar electromagnetic sensor," in Instrumentation and Measurement Technology Conference (I2MTC), 2011 IEEE, 2011, pp. 1-6.

[22] M. A. M. Yunus, S. Mukhopadhyay, and A. Punchihewa, "Application of independent component analysis for estimating nitrate contamination in natural water sources using planar electromagnetic sensor," in Sensing Technology (ICST), 2011 Fifth International Conference on, 2011, pp. 538-543.

[23] M. A. M. Yunus and S. C. Mukhopadhyay, "Novel planar electromagnetic sensors for detection of nitrates and contamination in natural water sources," Sensors Journal, IEEE, vol. 11, pp. 1440-1447, 2011

[24] M. A. M. Yunus, S. C. Mukhopadhyay, and S. Ibrahim, "Planar electromagnetic sensor based estimation of nitrate contamination in water sources using independent component analysis," Sensors Journal, IEEE, vol. 12, pp. 2024-2034, 2012.

[25] M. A. M. Yunus and S. Mukhopdhyay, "A new method for monitoring ammonium nitrate contamination in natural water sources based on independent component analysis," in Sensors, 2011 IEEE, 2011, pp. 1066-1069.

[26] A. Mohd Syaifudin, M. Yunus, S. Mukhopadhyay, and K. Jayasundera, "A novel planar interdigital sensor for environmental monitoring," in Sensors, 2009 IEEE, 2009, pp. 105-110.

[27] A. Rahman and M. S. Bin, "Novel planar interdigital sensors for the detection of bacterial endotoxins: a thesis presented in partial fulfilment of the requirements for the degree of Doctor of Philososphy in Electronics Engineering at Massey University, Palmerston North, New Zealand," 2012.

[28] S. C. Mukhopadhyay and C. P. Gooneratne, "A novel planar-type biosensor for noninvasive meat inspection," Sensors Journal, IEEE, vol. 7, pp. 1340-1346, 2007.

[29] K. Sundara-Rajan, L. Byrd, and A. V. Mamishev, "Moisture content estimation in paper pulp using fringing field impedance spectroscopy," Sensors Journal, IEEE, vol. 4, pp. 378-383, 2004.

[30] Y. Natsume and H. Sakata, "Zinc oxide films prepared by sol-gel spin-coating," Thin solid films, vol. 372, pp. 30-36, 2000.

[31] Y. Lu, R. Ganguli, C. A. Drewien, M. T. Anderson, C. J. Brinker, W. Gong, et al., "Continuous formation of supported cubic and hexagonal mesoporous films by sol-gel dip-coating," Nature, vol. 389, pp. 364-368, 1997.

[32] R. A. Caruso, J. H. Schattka, and A. Greiner, "Titanium dioxide tubes from sol-gel coating of electrospun polymer fibers," Advanced Materials, vol. 13, pp. 1577-1579, 2001.

[33] B. Wang, L. Zhu, Y. Yang, N. Xu, and G. Yang, "Fabrication of a $\mathrm{SnO} 2$ nanowire gas sensor and sensor performance for hydrogen," The Journal of Physical Chemistry C, vol. 112, pp. 6643-6647, 2008.

[34] Y.-J. Choi, I.-S. Hwang, J.-G. Park, K. J. Choi, J.-H. Park, and J.$\mathrm{H}$. Lee, "Novel fabrication of an $\mathrm{SnO} 2$ nanowire gas sensor with high sensitivity," Nanotechnology, vol. 19, p. 095508, 2008.

[35] A. Lasia, Electrochemical impedance spectroscopy and its applications: Springer, 2014

[36] B. Ruhland, T. Becker, and G. Müller, "Gas-kinetic interactions of nitrous oxides with SnO 2 surfaces," Sensors and Actuators B. Chemical, vol. 50, pp. 85-94, 1998.

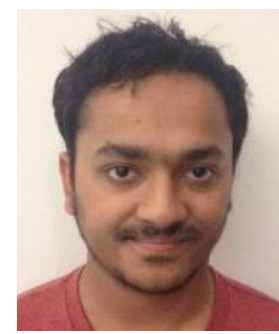

Mr. Anindya Nag has completed Bachelor of Technology from WEST BENGAL UNIVERSITY OF TECHNOLOGY in 2013 and Master of Engineering at Massey University, Palmerston North, New Zealand in June 2015. His research interests are in the area of Smart Sensors and Sensing Technology for home and environmental monitoring. $\mathrm{He}$ is currently pursuing $\mathrm{PhD}$ in Engineering at Massey University, Palmerston North, New Zealand and is working on Printable Electronic Sensors for Human Wellness.

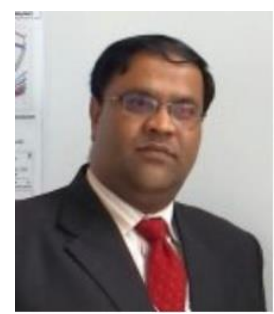

Dr. Asif Iqbal Zia (M'14) received the B.Sc. degree in electronics and the M.Sc. degree in physics from the University of the Punjab, Lahore, Pakistan, in 1990 and 1992, respectively. $\mathrm{He}$ has completed $\mathrm{PhD}$ in Engineering from Massey University, New Zealand in January 2015. He is currently working on physical electrochemistry with current interests in the development of chemical and biochemical sensors, electrochemical impedance spectroscopy, and molecular imprinting. He has authored 12 research articles in different international journals and conferences.

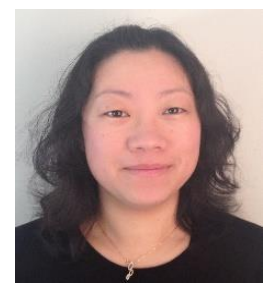

Mrs. Xie Li has completed Bachelor of Engineering from Massey University in 2014. She is currently working towards her Master of Engineering degree at the School of Engineering and Advanced Technology, Massey University, New Zealand and working on the development of a trial Nitrate sensor for practical application.

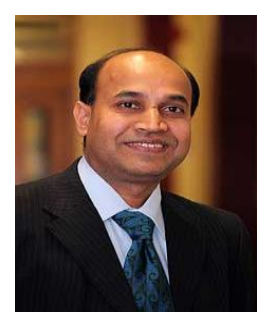

Dr. Subhas Chandra Mukhopadhyay (M'97, SM'02, F'11) is working as a Professor of Sensing Technology with the School of Engineering and Advanced Technology, Massey University, Palmerston North, New Zealand. He is a Fellow of IEEE (USA), a Fellow of IET (UK). He is a Topical Editor of IEEE Sensors journal, an Associate Editor of IEEE Transactions on Instrumentation and Measurements and a Technical Editor of IEEE Transactions on Mechatronics. He was a Distinguished Lecturer of IEEE Sensors Council from 2010-2013. He chairs the IEEE IMS Technical Committee 18 on Environmental Measurements.

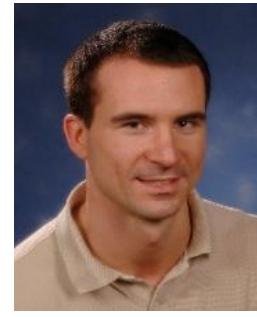

Dr. Jurgen Kosel is Associate Professor of Electrical Engineering at the King Abdullah University of Science and Technology (KAUST), Thuwal, Saudi Arabia, and head of the Sensing, Magnetism and Microsystems Research Group. He was a post-doctoral fellow with the Biomedical Engineering Research Group, Stellenbosch University, South Africa, from 2007 to 2009. From 2006 to 2007, he worked in the automotive industry as a Project Manager with Magna Powertrain, Graz, Austria. He received the Dipl.-Ing. (M.Sc.) and $\mathrm{PhD}$ degrees in electrical engineering from the Vienna University of Technology, Vienna, Austria, in 2002 and 2006, respectively. His research interests are in the fields of micro and nano devices with a focus on magnetic transducers. 